\title{
Few-cycle laser-pulse collapse in Kerr media: The role of group-velocity dispersion and $\mathrm{X}$-wave formation
}

\author{
Daniele Faccio, ${ }^{1,4}$ * Matteo Clerici, ${ }^{1,4}$ Alessandro Averchi, ${ }^{1,4}$ Antonio Lotti, ${ }^{1,4}$ Ottavia Jedrkiewicz, ${ }^{1,4}$ Audrius Dubietis, ${ }^{2}$ \\ Gintaras Tamosauskas, ${ }^{2}$ Arnaud Couairon, ${ }^{3,4}$ Francesca Bragheri, ${ }^{5}$ Dimitris Papazoglou, ${ }^{6,7}$ \\ Stelios Tzortzakis, ${ }^{7}$ and Paolo Di Trapani ${ }^{1,2,4}$ \\ ${ }^{1}$ CNISM and Department of Physics and Mathematics, Università dell'Insubria, Via Valleggio 11, IT-22100 Como, Italy \\ ${ }^{2}$ Department of Quantum Electronics, Vilnius University, Sauletekio Ave. 9, bldg. 3, LT-10222, Vilnius, Lithuania \\ ${ }^{3}$ Centre de Physique Théorique, CNRS, École Polytechnique, F-91128 Palaiseau, France \\ ${ }^{4}$ Virtual Institute for Nonlinear Optics, Centro di Cultura Scientifica Alessandro Volta, \\ Villa Olmo, Via Simone Cantoni 1, 22100 Como, Italy \\ ${ }^{5}$ Department of Quantum Electronics, University of Pavia, Pavia \\ ${ }^{6}$ Materials Science and Technology Department, University of Crete, P.O. Box 2208, 71003, Heraklion, Greece \\ ${ }^{7}$ Institute of Electronic Structure and Laser, Foundation for Research and Technology Hellas, P.O. Box 1527, 71110, Heraklion, Greece
}

(Received 5 May 2008; published 18 September 2008)

\begin{abstract}
We study ultrashort laser-pulse propagation and filamentation dynamics in dispersive Kerr media. We identify the regime for which the filamentation threshold $\left(P_{\mathrm{th}}\right)$ is considerably higher than the critical power $\left(P_{\mathrm{cr}}\right)$ for monochromatic beam collapse in pure Kerr media. In particular, we compare the threshold for the formation of filaments with that for the formation of $\mathrm{X}$-waves. At powers $P_{\mathrm{cr}}<P<P_{\mathrm{th}}$, self-focusing is arrested by group-velocity dispersion, and no filaments or $\mathrm{X}$-waves are formed. At $P \geqslant P_{\text {th }}$, we observe $\mathrm{X}$-wave formation and a weak filamentation regime. At $P \gg P_{\text {th }}$, we observe both $\mathrm{X}$-waves and fully formed filaments.
\end{abstract}

DOI: 10.1103/PhysRevA.78.033826

PACS number(s): 42.65.Jx, 42.65.Re

\section{INTRODUCTION}

Ultrashort laser-pulse filamentation is proving to be extremely important for our understanding of fundamental nonlinear laser-matter interactions and also for applications ranging from white light sources [1] and few-cycle pulse generation $[2,3]$ to efficient frequency conversion $[4,5]$. Important applications are also related to lightning protection [6-9], remote LIDAR sensing of chemical elements in the atmosphere $[10,11]$, and remote $\mathrm{THz}$ spectroscopy $[12,13]$. It is common use to refer to a critical threshold power defined for two-dimensional (2D) self-focusing of a monochromatic Gaussian beam $P_{\mathrm{cr}}=1.836 \lambda^{2} /\left(4 \pi n_{0} n_{2}\right)$ [16], where $n_{0}$ and $n_{2}$ are the linear and nonlinear indexes, respectively. For sufficiently long pulses when the input pulse power is larger than $P_{\mathrm{cr}}$, self-focusing will dominate over diffraction and the input pulse will collapse, as predicted more than 40 years ago [14]. The collapse may be arrested by various mechanisms such as multiphoton absorption (MPA) or self-induced plasma defocusing [17]. Beyond the point of tightest focus, which is referred to as nonlinear focus, the beam then evolves into a filament, i.e., a tightly focused peak that maintains a very small diameter over many diffraction lengths. A fundamental parameter of the input laser pulse is the duration $\tau$. Long, many-ps pulses will unavoidably lead to avalanche ionization, material damage, and very short filament lengths. The first long-range filaments were observed in air with 200 -fs-long input pulses [15], and continuous advances in laser technology now render few-cycle laser pulses commercially available. Due to the importance of the pulse duration in the filamentation process, it is necessary to understand

\footnotetext{
*daniele.faccio@uninsubria.it
}

how the various effects and in particular group velocity dispersion (GVD) come into play. Indeed, it is important to underline that the critical power $P_{\mathrm{cr}}$ is defined for a monochromatic beam and therefore neglects any possible effects related to the temporal dynamics of a pulse. It is well known that if the pulse is short enough, then self-focusing may be slowed down and eventually arrested by pulse temporal broadening due to GVD and splitting $[18,19]$.

Filamentation has been interpreted in terms of spontaneous formation of conical waves, in particular of $\mathrm{X}$-waves $[20,21]$. These are stationary states in both linear and nonlinear regimes and are characterized by an intense central peak in the near field, which may be identified with the filament core, and by a marked angularly dispersed spectral broadening in the Fourier domain, often referred to as conical emission (CE). It has been shown that in condensed media with relatively low self-generated plasma densities, the conical waves are generated at the nonlinear focus of the collapsing pulse where MPA plays a major role in reshaping the input Gaussian pulse into a conical wave [22]. More recently, the description of filaments in terms of $\mathrm{X}$-waves has been extended to filamentation in gaseous media such as air in which the collapse is arrested by plasma defocusing [23]. The question concerning the role played by $\mathrm{X}$-waves during filamentaion is still open. Particularly, it is worthwhile to clarify if they are inherent to the filamentation process, or if they are just side products that often accompany filamentation.

In this paper, we investigate both experimentally and numerically the filamentation threshold in condensed media in the regime of ultrashort 20-40 fs laser pulses. We show that the power threshold, previously identified as that above which GVD is not able to arrest collapse [19], coincides with both the filamentation threshold and the generation of $\mathrm{X}$-waves and associated features, e.g., a conical energy flux, 


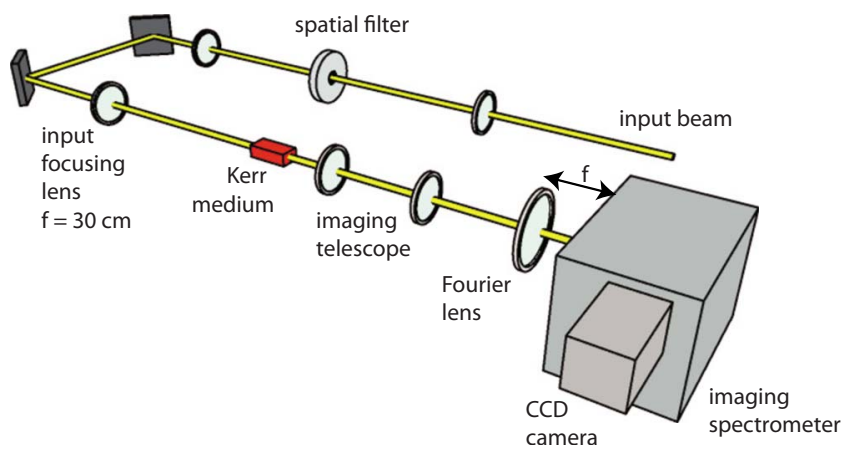

FIG. 1. (Color online) Experimental layout used for the measurements.

so that $\mathrm{X}$-waves have to be considered as structural to the filament and that one cannot exist without the other.

\section{EXPERIMENTS}

Experiments were performed using $25 \mathrm{fs}$ pulses at $700 \mathrm{~nm}$ delivered by a noncollinear optical parametric amplified (Topas white, light Conversion Ltd., Vilnius, Lithuania) pumped by an amplified Ti:sapphire laser. The experimental layout is shown in Fig. 1. The pulses were first spatially filtered and then focused onto a $4 \mathrm{~cm}$ sample of water with a $30 \mathrm{~cm}$ focal length lens. The output spectra were measured with a home-built imaging spectrometer with the input slit placed in the focal plane of a $10 \mathrm{~cm}$ lens and recorded with a modified Nikon D70 digital camera so as to obtain the $(\theta, \lambda)$ angularly resolved spectrum. Figures 2(a)-2(d) show the measured spectra with increasing input energy. The input spectrum (a) splits into two separate spectral components (b). This is followed by the development of conical emission that spreads out from the two spectral peaks with the notable feature that the redshifted (blueshifted) peak originates a blueshifted (redshifted) CE, so that the CE tails intersect close to $700 \mathrm{~nm}$, at $\sim \pm 20 \mathrm{mrad}$. In keeping with the $\mathrm{X}$-wave interpretation of filamentation, we may fit the spectrum in Fig. 2(c) using the relation $k_{\perp}=\sqrt{k^{2}-k_{z}^{2}}$ with $k$

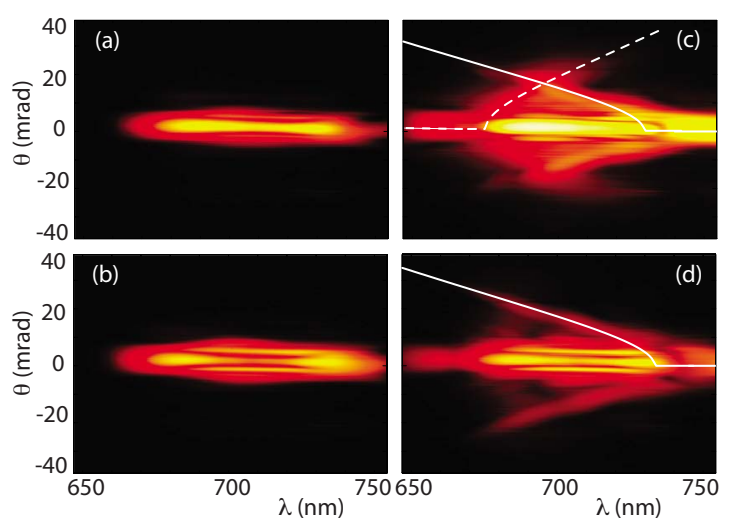

FIG. 2. (Color online) Experimental spectra measured after a 4-cm-long water sample with an input pulse centered at $700 \mathrm{~nm}$ and with increasing energy: (a) $300 \mathrm{~nJ}$, (b) $500 \mathrm{~nJ}$, (c) $600 \mathrm{~nJ}$, and (d) $800 \mathrm{~nJ}$.

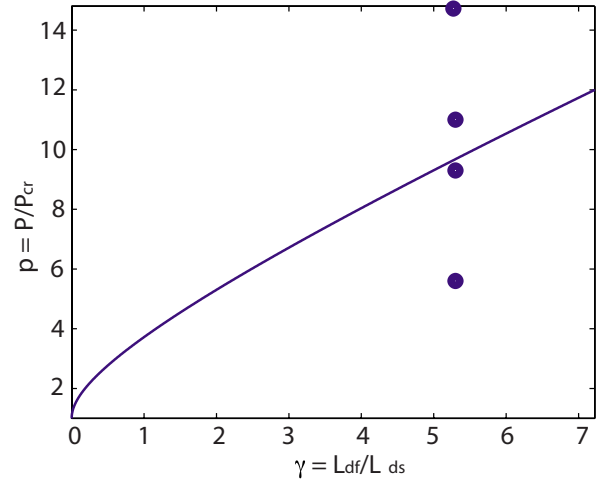

FIG. 3. (Color online) Solid line: power, $P_{\text {th }}$ (normalized with respect to $P_{\mathrm{cr}}$ ) above which beam collapse will occur according to the relation given in Ref. [19]. Dots: input pulse powers, corresponding to input energies of $300,500,600$, and $800 \mathrm{~nJ}$, investigated numerically and experimentally in this work.

$=(\omega / c) n(\omega)$ and $k_{z}(\omega)=k\left(\omega_{0}\right)+\left(\omega-\omega_{0}\right) / v_{g}$. The group velocity $v_{g}$ of the $\mathrm{X}$-wave may be determined directly from the $(\theta, \lambda)$ spectrum as described elsewhere $[5,24,25]$ : the two CE features give two separate $v_{g}$ values. The dashed line shows the $\mathrm{X}$-wave relation obtained with a value of $v_{g}$ $=2.2335 \pm 0.0003 \times 10^{8} \mathrm{~m} / \mathrm{s}$ while the solid line is obtained with $v_{g}=2.227 \pm 0.0002 \times 10^{8} \mathrm{~m} / \mathrm{s}$. This shows that the $\mathrm{CE}$ patterns correspond to two separate $\mathrm{X}$-waves that propagate slightly faster (slower) than the input Gaussian pulse $\left(v_{g}\right.$ $\left.=2.23 \times 10^{8} \mathrm{~m} / \mathrm{s}\right)$. In the near field this corresponds to a splitting of the on-axis intensity peak [20,21]. At even higher input energies, Fig. 2(d), the faster $\mathrm{X}$-wave strengthens considerably at the expense of the slower $\mathrm{X}$-wave, which is hardly visible. If we compare these spectra to those measured with longer pulses (see, for example, Refs. [5,20]), we immediately note the strong spectral splitting of the two X-waves: these usually pass through, or very close to, the same central pump wavelength while the measurements with 25 fs pulses clearly highlight that GVD is playing an important role which, together with the self-focusing nonlinearity, leads to a spectral splitting of the axial spectrum. Indeed for decreasing input pulse durations we may expect GVD to play an increasingly important role in the dynamics that lead to filament formation. Luther et al. have studied numerically the various regimes in which self-focusing or pulse broadening will dominate. In the framework of a model that accounts only for diffraction, dispersion, and Kerr nonlinear response, they have provided an analytical relation that allows us to distinguish between one and the other depending on input pulse duration, beam width, and intensity [19]. A parameter $\gamma$ is defined as the ratio between the pulse diffraction length $L_{\mathrm{df}}=k w_{0}^{2} / 2$ and dispersion length $L_{\mathrm{ds}}=\tau^{2} / k^{\prime \prime}$, where $w_{0}$ is the pulse radius, $k$ is the wave vector, and $k^{\prime \prime}=d^{2} k / d \omega^{2}$ is the GVD parameter. Their results show that a threshold power exists, $P_{\text {th }}$, above which the nonlinear dynamics always leads to collapse. $P_{\text {th }}$, however, increases with gamma. This means that for very short pulses, a sizable gap between $P_{\text {th }}$ and $P_{\mathrm{cr}}$ occurs. The above-mentioned analytical relation is shown as a solid line in Fig. 3. For powers $P_{\mathrm{cr}}<P<P_{\mathrm{th}}$, where $P_{\mathrm{th}}$ is a threshold power, the pulse will self-focus and collapse will be arrested by GVD. For $P>P_{\text {th }}$, the regime is self-focusing 


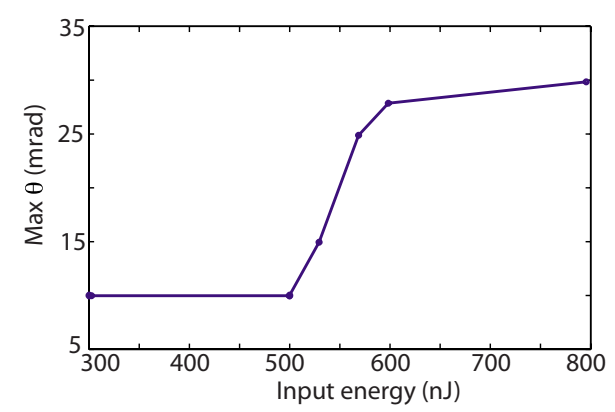

FIG. 4. (Color online) Experimentally measured maximum divergence angle of emitted radiation versus input energy. Each point is determined within a 2 mrad indetermination.

dominated and collapse occurs. For the parameters of our experiment, we find $\gamma=5.25$ and $P_{\mathrm{th}}=9.6 P_{\mathrm{cr}}$. The actual input powers in Figs. 2(a)-2(d) correspond to $P=5.6,9.3,11$, and $14.8 \times P_{\text {cr }}$, respectively (solid circles in Fig. 3), so that we are effectively studying the transition from the GVDdominated regime to the self-focusing dominated regime.

This threshold-like behavior is shown in more detail in Fig. 4, where we plot the measured maximum divergence angle of the laser pulse exiting the Kerr medium for varying input pulse energy. As may be seen, close to the predicted threshold energy of $500 \mathrm{~nJ}\left(9.3 P_{\mathrm{cr}}\right)$ the divergence angle increases sharply and then levels off. The observed sharp increase in $\theta$ therefore gives an indication regarding the threshold nature of this process. This increase is related to the formation of X-tails in the spectrum, i.e., to the development of conical emission, which is known to be an indication of the spontaneous formation of $\mathrm{X}$-waves. As we will show in the following section, the same threshold behavior is observed also in the formation of the filament, thus linking $\mathrm{X}$-waves and filaments not only through their simultaneous appearance but also through their abrupt formation dynamics.

\section{NUMERICS}

In order to gain a deeper insight into the physics of the pulse collapse process in the regime of strong GVD broadening, we performed a series of numerical simulations under conditions similar to the experiments.

The code used has been described in detail elsewhere [17] and here we recall only its general features outlining the separate terms used and that are of importance for this work. We solve the nonlinear equation for the envelope $\mathcal{E}_{\omega, r, z}=\mathcal{F}\left(\mathcal{E}_{t, r, z}\right)$, where $\mathcal{F}$ denotes Fourier transform and the laser field with central frequency $\omega_{0}$ reads $E_{t, r, z}$ $=\mathcal{E}_{t, r, z} \exp \left(-i \omega_{0} t+i k_{0} z\right)$. The derivation of the envelope equation from the wave equation in nonlinear media is recalled in Refs. [17,26],

$$
\begin{aligned}
2 K_{g} \frac{\partial \mathcal{E}_{\omega, r, z}}{\partial z}= & i \nabla_{\perp}^{2} \mathcal{E}_{\omega, r, z}+i\left[k^{2}(\omega)-K_{g}^{2}\right] \mathcal{E}_{\omega, r, z} \\
& +\left(i \frac{\omega^{2}}{c^{2}} \mathcal{P}_{\omega, r, z}-\frac{\omega}{c^{2}} \mathcal{J}_{\omega, r, z}\right)
\end{aligned}
$$

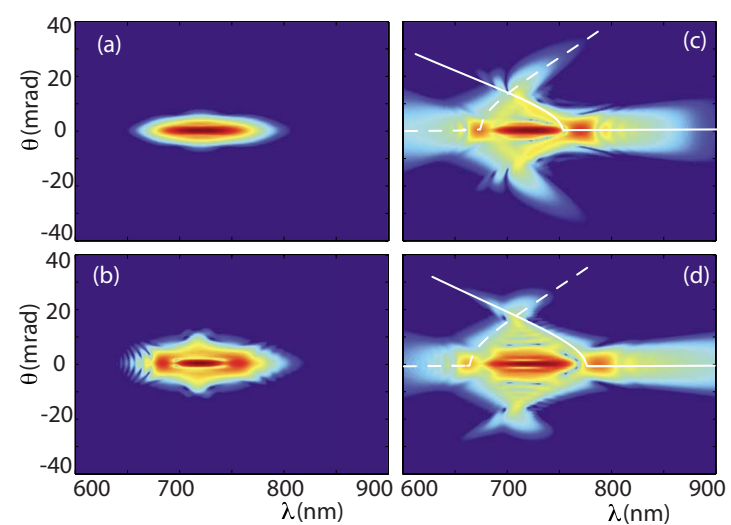

FIG. 5. (Color online) Numerical spectra in logarithmic scale (7 decades) centered at $700 \mathrm{~nm}$ and with increasing energy: (a) $300 \mathrm{~nJ}$, (b) $500 \mathrm{~nJ}$, (c) $600 \mathrm{~nJ}$, and (d) $800 \mathrm{~nJ}$.

where $K_{g}=k_{0}+k_{0}^{\prime}\left(\omega-\omega_{0}\right)$. The first two terms on the righthand side (r.h.s.) of Eq. (1) account for diffraction in the transverse plane and dispersion described via the Sellmeier relation $k(\omega)$ given by Eq. (12) in Ref. [28], which defines the wave number $k_{0} \equiv k\left(\omega_{0}\right)$, the group velocity $1 / k_{0}^{\prime}$ $\equiv 1 /\left.(\partial k / \partial \omega)\right|_{\omega_{0}}$, and the refraction index $n_{0}=k_{0} c / \omega_{0}$. The polarization $P_{t, r, z}=\mathcal{P}_{t, r, z} \exp \left(-i \omega_{0} t+i k_{0} z\right)$ describes the optical Kerr effect with coefficient $n_{2}$. It is split into an instantaneous component and a delayed (Raman-Kerr) component with fraction $f_{R}$. The polarization envelope $\mathcal{P}_{t, r, z}$ is expressed as

$$
\begin{gathered}
\mathcal{P}_{t, r, z}=2 n_{0} n_{2}\left[\int_{-\infty}^{t} d t^{\prime} R\left(t-t^{\prime}\right)\left|\mathcal{E}_{t^{\prime}, r, z}\right|^{2}\right] \mathcal{E}_{t, r, z}, \\
R(t)=\left(1-f_{R}\right) \delta(t)+f_{R} \frac{\Lambda^{2}+\Gamma^{2}}{\Lambda} \mathrm{e}^{-\Gamma t} \sin (\Lambda t) .
\end{gathered}
$$

Multiphoton processes and plasma induced phenomena are described via the current and the evolution equation for the electron density,

$$
\begin{gathered}
\mathcal{J}=c\left[\sigma(\omega) \rho \mathcal{E}-n_{0} \beta_{K}|\mathcal{E}|^{2 K-2} \mathcal{E}\right], \\
\sigma(\omega)=\sigma_{r}+i \sigma_{i}=\frac{\omega_{0}}{c \rho_{c}} \frac{\omega_{0} \tau_{c}\left(1+i \omega \tau_{c}\right)}{1+\omega^{2} \tau_{c}^{2}}, \\
\frac{\partial \rho}{\partial t}=\frac{\beta_{K}}{K \hbar \omega_{0}}|\mathcal{E}|^{2 K}+\frac{\sigma_{r}}{U_{i}}|\mathcal{E}|^{2},
\end{gathered}
$$

where $\beta_{K}$ denotes the multiphoton absorption cross section, $K$ is the number of photons involved in the process, $U_{i}$ is the ionization potential of the medium, $\tau_{c}$ is the collision time for the inverse bremsstrahlung process, and $\rho_{c}$ is the plasma density above which the medium becomes opaque at $\omega_{0}$.

Figures 5(a)-5(d) show the numerically calculated spectra for the same input conditions of the experimental results in Fig. 2. In particular, Figs. 5(a)-5(c) were performed including only the first three terms on the r.h.s. of Eq. (1) and with $f_{R}=0$ (i.e., accounting only for diffraction, dispersion, and instantaneous Kerr nonlinearity). Input energies higher than 


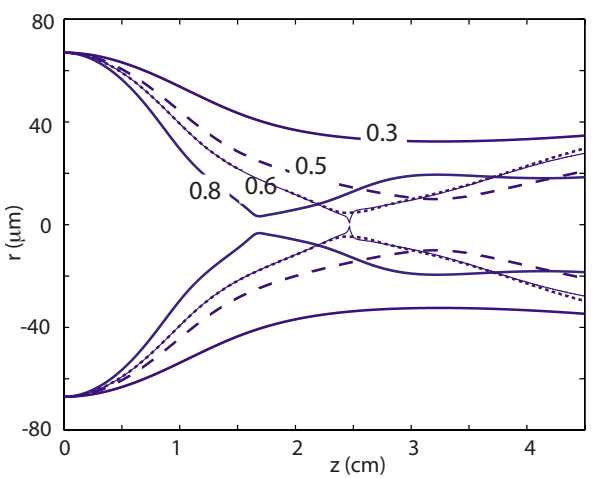

FIG. 6. (Color online) Numerically calculated beam radii at FWHM for the same conditions as in Fig. 5. The input pulse energies are indicated on the relative curves.

$0.6 \mu \mathrm{J}$ required the use of the full equation in order to avoid numerical divergence. The results reproduce the experimental findings and in particular the CE features. Figure 6 shows the beam radii at full width at half-maximum (FWHM) for the four input energies studied in Figs. 2 and 5: at low input energies $(0.3$ and $0.5 \mu \mathrm{J})$ the beam undergoes a slight selffocusing but collapse is quickly arrested by GVD and relatively low peak intensities $\left(40\right.$ and $\left.100 \mathrm{GW} / \mathrm{cm}^{2}\right)$ are reached at the tightest focus point. We note that for these energies, we observed no difference in the simulations when including all nonlinear terms (e.g., multiphoton absorption and plasma generation) or when including only diffraction, dispersion, and self-focusing. For an input energy of $0.6 \mu \mathrm{J}$ we are in the regime $P \simeq P_{\text {th }}$ yet GVD alone is still able to arrest the collapse. However, the FWHM curve (thin solid line in Fig. 6) shows a cusp at the nonlinear focus which is due to collapse arrested by the finite numerical grid. In order to obtain a more meaningful result, we repeated the simulation including also multiphoton absorption and we observed a smoother behavior in the pulse evolution close to the nonlinear focus (short-dashed line). This indicates that NLLs are starting to play a non-negligible role in the pulse evolution. Furthermore, we note that the pulse propagation after the nonlinear focus may not be described in terms of simple linear Gaussian-beam diffraction due to the fact that the peak is strongly subdiffractive, i.e., the FWHM increases at a much slower rate (about $5 \times$ slower) than that expected for a Gaussian beam with the same FWHM diameter. We therefore identify this regime as a "weak" filament.

For higher energies, we were not able to arrest the pulse collapse with GVD alone and we may conclude that indeed the pulse is in the self-focusing dominated regime [31]. For $0.8 \mu \mathrm{J}$ input energy, the FWHM evolution after the nonlinear focus is no longer monotonic due to continued nonlinear interactions: we may identify this regime as a "fully formed" filament.

We now consider the role of $\mathrm{X}$-wave formation in filamentation. Indeed it is known that $\mathrm{X}$-waves are stationary solutions in the presence of a self-focusing nonlinearity and GVD and that these are spontaneously generated within filaments. X-waves are characterized by a number of features that allows them to be identified:

(i) In the far-field, the $\mathbf{X}$-wave spectrum is $\mathbf{X}$-shaped, i.e., formed by hyperbolic tails that have a well-defined $\theta=\theta(\lambda)$ dependence, as described above.

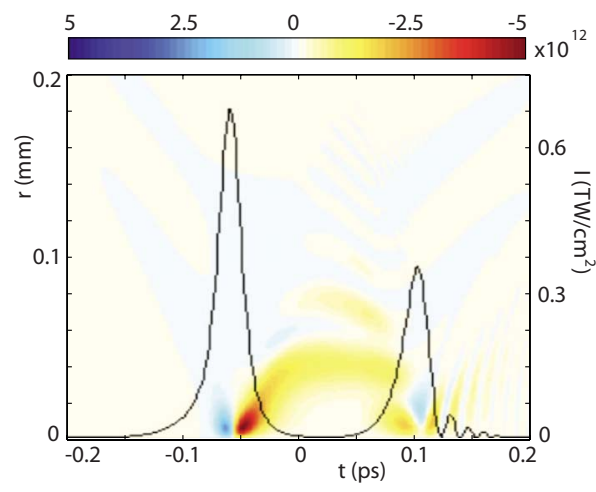

FIG. 7. (Color online) Numerically calculated energy density flux near-field distribution for an input energy of $0.6 \mu \mathrm{J}$, at a propagation distance of $3 \mathrm{~cm}$. The color map is in arbitrary units and the solid black line shows the on-axis temporal intensity profile of the pulse (right-hand axis).

(ii) In the near-field, the $\mathrm{X}$-wave is characterized by an intensity peak surrounded by slowly decaying tails, i.e., by an energy reservoir that continuously refills the peak, as highlighted also by a radial conical energy flux [23,29]. Moreover, the intensity peak exhibits a strongly subdiffractive evolution. Due to the finite energy contained in the surrounding energy reservoir, the subdiffractive peak will be maintained only over a finite distance.

All of these features may be found also in filaments and are considered as characteristic traits also of the filament. It is therefore interesting to try and understand what really constitutes a filament [30] and, consequently, the eventual role of $\mathrm{X}$-waves in sustaining the filament itself.

We consider the case of $0.6 \mu \mathrm{J}$ input energy, i.e., at the threshold for filament formation. If we look at the FWHM evolution along propagation (Fig. 6), we note that it is characterized by an initial focusing stage followed by subdiffractive defocusing that we associated with the formation of a weak filament. The corresponding spectrum in Fig. 5(c) shows clear CE, which indeed hints toward the formation of $\mathrm{X}$-waves. As for the near-field, it has been pointed out that self-focusing dynamics lead to complicated interference patterns that are difficult to interpret [20] and do not allow to readily isolate $\mathrm{X}$-wave features. This difficulty may be partially overcome by studying the near-field distribution of the transverse energy density flux rather than that of the actual electric field $[23,29]$. The rationale behind this approach is that the surrounding, low intensity reservoir is not strongly reshaped and does not exhibit a marked inward radial flux. Conversely, the central high-intensity region suffers strong spatio-temporal reshaping with a corresponding radial flux that therefore strongly separates it from the reservoir. This is shown in Fig. 7: the black solid line represents the on-axis temporal intensity profile of the pulse. The input pulse has split in two and the flux corresponding to each pulse and in particular to the leading pulse shows a strong conical (Vshaped in the $r>0$ plane) distribution with an inward (outward) flux on the leading (trailing) edge. This conical flux therefore confirms that indeed the input Gaussian pulse has reshaped into a conical wave, in particular into an X-wave. As a further confirmation of this fact, we study the evolution 


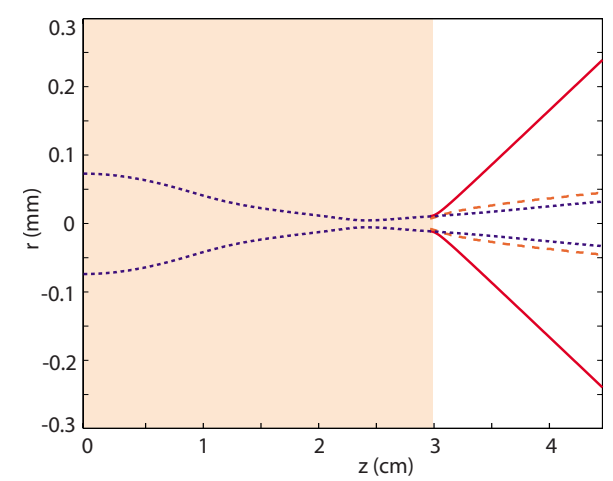

FIG. 8. (Color online) Dotted line-same as in Fig. 6 with input energy $0.6 \mu \mathrm{J}$. Dashed line-at $3 \mathrm{~cm}$ all nonlinear effects are switched off and further propagation is purely linear. Solid lineexpected Gaussian linear diffraction for a beam with the same width of the filament peak. The shaded area indicates the propagation region in which all nonlinear effects are included.

of the pulse FWHM after the propagation distance $(3 \mathrm{~cm})$ considered in Fig. 7. Figure 8 shows the FWHM evolution when all nonlinear effects are included over the full $4.4 \mathrm{~cm}$ propagation distance (blue dotted line). The red dashed line corresponds to the numerically calculated evolution when, at a distance $z=3 \mathrm{~cm}$, all nonlinear effects are switched off and purely linear propagation ensues. The solid red line shows the expected FWHM evolution for a Gaussian beam that has the same FWHM of the pulse peak at $z=3 \mathrm{~cm}$. As may be seen, the actual numerically calculated evolution of the pulse exiting from the nonlinear propagation regime is highly subdiffractive and is nearly identical to that of the pulse with all nonlinear effects switched on. Clearly this subdiffractive propagation has nothing to do with the material nonlinearity or nonlinear balancing effects and may only be ascribed to the particular conical nature of the pulse itself. These results lead us to the conclusion that spatiotemporal reshaping occurs close to the nonlinear focus and that the ensuing (weak) filament is nothing more than the propagation of the resulting $\mathrm{X}$-wave(s).

Increasing the input energy does not qualitatively modify any of the above results. A conical energy flux is associated with each of the splitted pulses and strongly subdiffractive propagation is still observed after the nonlinear focus even if all nonlinear effects are switched off. However, quantitative differences are observed: as the input power is increased above the threshold for the self-focusing dominated regime, both the peak intensity reached at the nonlinear focus and the conical energy flux increase by an order of magnitude or more with respect to that shown in Fig. 7. We give an example of this in Fig. 9, which shows the filament intensity and energy flux distributions for an input energy of $1.5 \mu \mathrm{J}$. A marked difference is observed also in the FWHM evolution, which may no longer be simply separated into a first focusing and a second defocusing stage but rather exhibits a nonmonotonic evolution in the second stage (e.g., see Fig. 6 at $0.8 \mu \mathrm{J}$ ) and will eventually show multiple refocusing stages, each characterized by a replenishment of energy toward the center of the pulse and successive splitting into daughter pulses $[20,27]$. Quantitative differences are observed also in

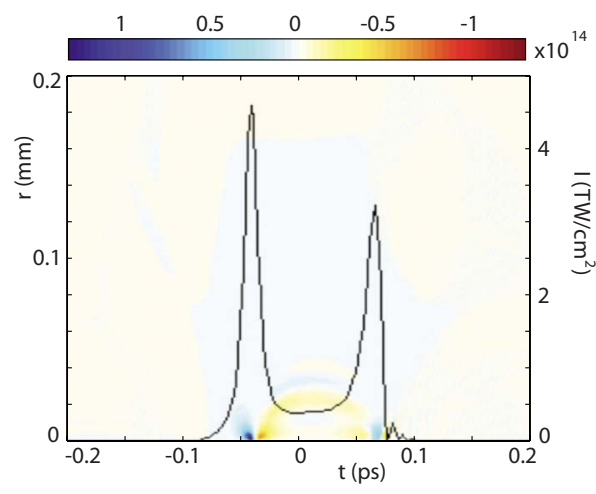

FIG. 9. (Color online) Numerically calculated energy density flux near-field distribution for an input energy of $1.5 \mu \mathrm{J}$, at a propagation distance of $1.125 \mathrm{~cm}$. The color map is in arbitrary units and the solid black line shows the on-axis temporal intensity profile of the pulse (right-hand axis).

the $(\theta, \lambda)$ spectrum: Fig. 10 shows the numerically calculated spectrum with $1.5 \mu \mathrm{J}$ input energy. With respect to Fig. 5 the $\mathrm{CE}$, i.e., the $\mathrm{X}$-wave spectrum, is now much more developed and shows well-defined tails that extend far away from the pump wavelength. We verified this behavior experimentally by sending a $35 \mathrm{fs}, 3 \mu \mathrm{J}, 800 \mathrm{~nm}$ pulse into a $4-\mathrm{cm}$-long sample of sapphire. As shown in Fig. 11, the spectrum, measured with a commercial imaging spectrometer (Shamrock, Andor), exhibits the main characteristics of Fig. 10. We also note that the traits outlined in this paragraph remain similar in all media (condensed or gaseous) and for all wavelengths within the normal GVD regime. In the self-focusing dominated regime, the much higher intensities lead to a much stronger nonlinear reshaping of the input Gaussian pulse. The stronger conical flux indicates more sharply defined $\mathrm{X}$-waves that also have high $\sim \mathrm{TW} / \mathrm{cm}^{2}$ peak intensities. The successive propagation is therefore dominated by a dynamical and nonlinear interaction between the splitted, highintensity $\mathrm{X}$-waves which generate the main features observed in filaments such as dynamical replenishment, $\mathrm{CE}$, ionization, and plasma generation.

\section{CONCLUSION}

In conclusion, we have examined the collapse and successive propagation dynamics of ultrashort laser pulses in non-

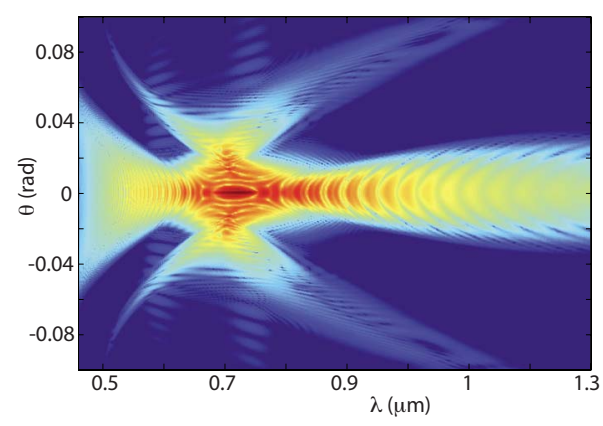

FIG. 10. (Color online) Numerical spectrum in logarithmic scale (10 decades) with an input energy of $1500 \mathrm{~nJ}$. 


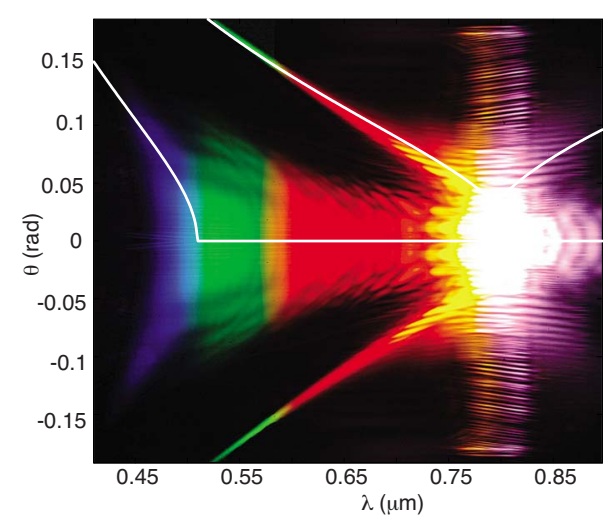

FIG. 11. (Color online) Experimental spectrum obtained with $3 \mu \mathrm{J}$ input energy, $35 \mathrm{fs}$ pulse duration and centered at $800 \mathrm{~nm}$ from a $4 \mathrm{~cm}$ sample of sapphire. The white solid lines indicate the best fits obtained using the $\mathrm{X}$-wave relation as described in the text.

linear Kerr media. For relatively long pulses, the collapsing pulse will be in the well known self-focusing dominated regime: beam collapse is arrested by a saturating mechanism, the nature of which (e.g., MPA, plasma defocusing, higherorder nonlinearity) will depend on the specific medium under study. Collapse arrest will be followed by filament propagation. This is the case, for example, of most experiments reported in the literature and applies to pulses with $\tau \gtrsim 50 \mathrm{fs}$ in condensed media or $\tau \gtrsim 10$ fs in air. Shorter pulses will suffer from strong GVD broadening, and this effect alone may arrest the collapse and no clear filament is observed. How- ever, as the input power is increased, a threshold power is reached below which self-focusing will dominate. For input powers close to this threshold, a weak filament is formed whose propagation is nearly indistinguishable from that of the linearly propagating $\mathrm{X}$-wave generated at the nonlinear focus. This gives a strong indication that the weak filament is nothing more than a linearly propagating $\mathrm{X}$-wave. For powers well above $P_{\mathrm{th}}$, much higher $\sim \mathrm{TW} / \mathrm{cm}^{2}$ intensities are reached and the well-known self-focusing dominated dynamics are recovered. The $\mathrm{X}$-waves will no longer propagate linearly or independently after the nonlinear focus but rather continue to interact through the instantaneous Kerr nonlinearity and eventually through delayed nonlinear effects such as stimulated Raman scattering and plasma generation.

\section{ACKNOWLEDGMENTS}

The authors acknowledge support from the Consorzio Nazionale Interuniversitario per le Scienze Fisiche della Materia (CNISM), project INNESCO, Access to Research Infrastructures activity in the Sixth Framework Programme of the EU (Contract No. RII3-CT-2003-506350, Laserlab Europe). P.D.T. acknowledges support from the Marie Curie Chair project STELLA MEXC-CT-2005-025710 and S.T. acknowledges support from the Marie Curie Excellence Grant MULTIRAD MEXT-CT-2006-042683. M.C. aknowledges the support from Sixth EU framework programme, Contract No. MEST-CF-2004-008048 (ATLAS) and MIUR, project no. RBIN04NYLH.
[1] R. R. Alfano, The Supercontinuum Laser Source (SpringerVerlag, New York, 1989).

[2] A. Couairon, J. Biegert, C. P. Hauri, W. Kornelis, F. W. Helbing, U. Keller, and A. Mysyrowicz, J. Mod. Opt. 53, 75 (2006).

[3] L. Gallmann, T. Pfeifer, P. M. Nagel, M. J. Abel, D. M. Neumark, and S. R. Leone, Appl. Phys. B: Lasers Opt. 86, 2503 (2007).

[4] F. Théberge, N. Aközbek, W. Liu, A. Becker, and S. L. Chin, Phys. Rev. Lett. 97, 023904 (2006).

[5] D. Faccio, A. Averchi, A. Couairon, M. Kolesik, J. V. Moloney, A. Dubietis, G. Tamosauskas, P. Polesana, A. Piskarskas, and P. Di Trapani, Opt. Express 15, 13079 (2007).

[6] X. M. Zhao and J. C. Diels, Laser Focus World 29, 113 (1993).

[7] S. Tzortzakis, B. Prade, M. Franco, A. Mysyrowicz, S. Hüller, and P. Mora, Phys. Rev. E 64, 057401 (2001).

[8] B. La Fontaine, D. Comtois, C.-Y. Chien, A. Desparois, F. Génin, G. Jarry, T. Johnston, J.-C. Kieffer, F. Martin, R. Mawassi, H. Pépin, F. A. M. Rizk, F. Vidal, C. Potvin, P. Couture, and H. P. Mercure, J. Appl. Phys. 88, 610 (2000).

[9] J. Kasparian, R. Ackermann, Y. B. Andrè, G. Méchain, G. Mèjean, B. Prade, P. Rohwetter, E. Salmon, K. Stelmaszczyk, J. Yu, A. Mysyrowicz, R. Sauerbrey, L. Wöste, and J. P. Wolf, Opt. Express 16, 5757 (2008).

[10] P. Rairoux, H. Schillinger, S. Niedermeier, M. Rodriguez, F.
Ronneberger, R. Sauerbrey, B. Stein, D. Waite, C. Wedekind, H. Wille, L. Wöste, and C. Ziener, Appl. Phys. B: Lasers Opt. 71, 573 (2000).

[11] J. Kasparian and J. P. Wolf, Opt. Express 16, 466 (2008).

[12] C. D’Amico, A. Houard, M. Franco, B. Prade, A. Mysyrowicz, A. Couairon, and V. T. Tikhonchuk, Phys. Rev. Lett. 98, 235002 (2007).

[13] C. D’Amico, A. Houard, S. Akturk, Y. Liu, J. Le Bloas, M. Franco, B. Prade, A. Couairon, V. T. Tikhonchuk, and A. Mysyrowicz, New J. Phys. 10, 013015 (2008).

[14] G. A. Askaryan, Sov. Phys. JETP 15, 1088 (1962).

[15] A. Braun, G. Korn, X. Liu, D. Du, J. Squier, and G. Mourou, Opt. Lett. 20, 73 (1995).

[16] R. Boyd, Nonlinear Optics (Academic, New York, 2002).

[17] A. Couairon and A. Mysyrowicz, Phys. Rep. 441, 47 (2007).

[18] P. Chernev and V. Petrov, Opt. Lett. 17, 172 (1992).

[19] G. G. Luther, J. V. Moloney, A. C. Newell, and E. M. Wright, Opt. Lett. 19, 862 (1994).

[20] M. Kolesik, E. M. Wright, and J. V. Moloney, Phys. Rev. Lett. 92, 253901 (2004).

[21] D. Faccio, M. A. Porras, A. Dubietis, F. Bragheri, A. Couairon, and P. Di Trapani, Phys. Rev. Lett. 96, 193901 (2006).

[22] A. Dubietis, E. Gaižauskas, G. Tamošauskas, and Paolo Di Trapani, Phys. Rev. Lett. 92, 253903 (2004).

[23] D. Faccio, A. Averchi, A. Lotti, P. Di Trapani, A. Couairon, D. Papazoglou, and S. Tzortzakis, Opt. Express 16, 1565 (2008). 
[24] D. Faccio, A. Averchi, A. Dubietis, P. Polesana, A. Piskarskas, P. Di Trapani, and A. Couairon, Opt. Lett. 32, 184 (2007).

[25] M. Kolesik, E. M. Wright, and J. V. Moloney, Opt. Express 13, 10729 (2005).

[26] A. Couairon, E. Gaižauskas, D. Faccio, A. Dubietis, and P. Di Trapani, Phys. Rev. E 73, 016608 (2006).

[27] M. Mlejnek, E. M. Wright, and J. V. Moloney, Opt. Lett. 23, 382 (1998).
[28] A. G. Van Engen, S. A. Diddams, and T. S. Clement, Appl. Opt. 37, 5670 (1998).

[29] P. Polesana, M. Franco, A. Couairon, D. Faccio, and P. Di Trapani, Phys. Rev. A 77, 043814 (2008).

[30] Y. Chen, F. Thèberge, O. Kosareva, N. Panov, V. P. Kandidov, and S. L. Chin, Opt. Lett. 32, 3477 (2007).

[31] A. Couairon, Phys. Rev. A 68, 015801 (2003). 